\title{
Aging in unity
}

\author{
We are excited to launch Nature Aging, a journal whose mission is to publish some of the most important and \\ timely research from across the entire aging research spectrum and be a nexus for the diverse communities \\ working on aging.
}

T he quest for eternal youth has long captured the imagination of humankind, but it's only in the past three decades that biology has established a scientific understanding of the aging process at the molecular and cellular level. These findings have not only provided a biological framework to understand aging, but they have also offered the promises of slowing down the physiological decline that comes with old age and preventing or treating age-associated diseases. Geroscience is starting to drive a paradigmatic shift in how we think about treating diseases and addressing the vulnerabilities in health that come with old age. This shift has recently materialized with the initiation of clinical trials that are attempting to intervene on age-related diseases in a holistic fashion, by acting on aging biology itself rather than modifying a single disease-specific process.

But aging research goes beyond deciphering aging biology in the laboratory and understanding and treating age-associated disorders in clinical settings. Aging research also aims to understand the impact of aging at the level of populations, with increasingly important public health and societal implications. According to the United Nations, the number of people aged 60 years old and older will double by 2050 , eventually reaching $22 \%$ of the world's population. Although humans live longer lives, many unfortunately don't live in good health later in life. Modern medicine has undeniably had a substantial impact on delaying mortality associated with major fatal diseases, but it has yet to substantially increase our healthspan: the length of life without disease or disability. Living good and healthy lives is not only about physical and cognitive health but also about mental and social wellbeing. A multigenerational society can only prosper if it is inclusive, and sadly, older adults are still too often being discriminated against because of their age, or being ostracized or even abused.

What transpires from this brief overview of what stands behind a single word is the complexity and diversity of all the questions related to aging that the research community is attempting to answer, as well as the importance of the challenges and the opportunities that lie ahead for our society to either address or seize. The anticipated benefits of the current and future research agendas and sets of societal policies on aging are huge and will affect us all. Everyone ages, and the progress we make now will have an impact on our future selves as well as on the future of our society. Healthy aging is one of the key societal priorities of the twenty-first century, which will require collective action and the pursuit of common goals across relevant research disciplines.

Political will informed by an evidence base is needed to enable societal change. The recent proclamation by the United Nations of the Decade of Healthy Ageing (2021-2030; http://bit.ly/3gXNBcW) has sent a strong signal that such political will exists at a global level. In health research, however, the disciplines that are pertinent to addressing and understanding aging remain fragmented due the existence of historical silos that continue to prevail. Traditionally, researchers from the biological, clinical, public health and social sciences rarely interact. Similarly, research on aging-related diseases tends to be compartmentalized, following the rule of one biological system or organ, one disease. While this organization of research has been successful in making important discoveries, the quest to understand aging and its relationship to disease as well as enabling the necessary societal changes to adapt to the ongoing demographic transformation would benefit from more interdisciplinary collaborations and discussions. In the words of the Scientific Director of the National Institute on Aging at the National Institutes of Health (see Q\&A in this issue), "we must learn to speak a common language".

Today, we are officially launching Nature Aging to contribute to this endeavor and support aging research in all its diversity. The journal aims to publish timely, high-profile research articles and reviews from all areas of the field, and provide a general discussion forum on aging in the form of comments and opinion pieces focused on scientific or societal questions. We seek to publish exciting aging research on the genetics, the molecular and cellular biology and the physiology of normal aging, but also preclinical and clinical studies on age-related diseases as well as research looking at the socioeconomic dimensions of human aging. We will also dedicate some of our pages to contributions coming from non-academic actors in the aging space, including members of industry and civil society. With this comprehensive scope and unique mission, we are creating a new, dedicated and visible platform for the diverse communities working on aging.

This first issue truly reflects the broad thematic goal of this journal by featuring primary research, reviews and opinion pieces authored by biologists, clinicians, social scientists and industry and civil society leaders. The topics covered include the impact of diets on aging and longevity; changes in human biology and physiology that take place in old age or across lifespans, such as in the immune or intestinal systems; conditions such as frailty, Alzheimer's disease and dementia; trends in life expectancies; long-term care policies; and the vulnerability of older adults to COVID19 and the challenges this has created for our healthcare systems. These themes, alongside our recently curated Nature Research online collection titled 'Aging, longevity and age-related diseases' (https:// www.nature.com/collections/dbagdcagdb), may serve as initial guidance for prospective authors, but we encourage you to contact us directly to discuss the suitability of potential submissions in more detail.

It is an exciting time to be working on aging. A strong biological foundation has been established and our understanding of aging processes is continuously developing. There is a plethora of stimulating new discoveries and initiatives that feed different streams of research in the lab, the clinic and the community. Crucially, there is an ever-growing motivation to address the challenges of global population aging and reimagine long-held views and social systems to seize the opportunities they present. We are thrilled to become a community partner for these efforts and look forward to witnessing what we expect to be the continued rise of a unified aging research field.

Published online: 14 January 2021

https://doi.org/10.1038/s43587-020-00022-2 